\title{
Linear response of the two-dimensional pure electron plasma: Quasimodes for some model profiles
}

\author{
Noel R. Corngold \\ California Institute of Technology, Pasadena, California 91125
}

(Received 26 September 1994; accepted 23 November 1994)

\begin{abstract}
After examining the initial value problem for the linear, diocotron response of a long cylinder of pure-electron plasma, the "quasimodes" associated with convex, power-law density profiles are studied. For these profiles, exact, analytic results are available. The "quasimodes," which are damped by phase mixing, may be characterized by their angular variation, flatness, and the magnitude of the gap separating the plasma from the containing wall. (c) 1995 American Institute of Physics.
\end{abstract}

\section{INTRODUCTION}

This paper is concerned with diocotron waves in a nonneutral plasma. In a recent experiment, ${ }^{\prime}$ Pillai and Gould excited the $m=2$, diocotron mode of a column of pureelectron plasma, and observed its subsequent, damped "ringing." When the amplitude of the short, exciting burst is relatively small, one expects a linear analysis of the response to be fruitful. (deGrassie and Malmberg, in an earlier study of diocotron waves, ${ }^{2}$ emphasized the nonlinear regime.) Linear "ringing" is usually associated with a normal mode of oscillation of a system, or with a discrete eigenvalue, or a point in the point spectrum of some operator describing the evolution of the system. But it has been known for some time ${ }^{3}$ that such modes do not exist in the collisionless dynamical model most commonly used to describe the pure-electron plasma, under the conditions believed to prevail in these experiments. There is, however, a continuous spectrum associated with the system, and it brings what appears to be a paradox: the evolution of a system governed by a continuous spectrum is generally nonexponential, but the experimenter sees exponential behavior. Two responses are available. One can simply remark that the integrand in the integration over the continuous range of frequencies is peaked sharply at the observed frequency. Or, one can ask why?-is the peak the "shadow" of an interesting singularity sitting nearby?-a "quasimode?" And might the quasimode be viewed as a signature, or used for diagnostics?

Of course, the situation is not new. It resembles that encountered in the study of Landau damping. To understand it fully one must-in the language of the theory-examine a multivalued Green's function which has been continued analytically onto a sheet adjacent to the "physical sheet." This problem was discussed generally and thoroughly, two decades ago, by Briggs, Daugherty, and Levy ${ }^{3}$ (henceforth BDL). This paper supplements BDL in showing that such an analysis may be carried out analytically for all angular modes-not merely $m=2$, or the peculiar $m=1$-in plasmas characterized by convex, power-law profiles. Then, the quantities of interest may be expressed in terms of hypergeometric functions and the continuation is straightforward. One finds the "quasimode," in an appropriate place. One obtains formulas for the dependence of frequency and damping of the mode upon profile, mode number, and gap size. To make our presentation coherent, we shall have to develop material that is presented either explicitly or implicitly in BDL. We hope that the repetition will not bore the reader. Finally, we note that the computations required to obtain specific numbers may be carried out easily with a hand calculator (one that does complex arithmetic!). We shall illustrate by considering three examples (cases): in the first the plasma fills the interior of a perfectly conducting cylindrical shell; in the second the plasma fills a "good," but not perfect shell, and in the third the plasma occupies the central portion of the interior of a perfect shell; a vacuum gap separates the plasma from the wall. This paper also contains some general discussion and some ideas about special solutions for profiles more complicated than power law, the latter being somewhat unnatural in their edge behavior.

\section{ANALYSIS}

The model we consider describes the plasma density $n(r, \theta, z)$ and the electrostatic potential $\phi(r, \theta, z)$ by the equations

$$
\frac{\partial n}{\partial t}+\frac{c}{B}(\nabla \phi \times \nabla n) \cdot \hat{z}=0, \quad \nabla^{2} \phi=-4 \pi q n .
$$

The plasma is contained in a very long, grounded circular cylinder, whose radius is denoted by " $a$." The system is immersed in an uniform magnetic field, $\mathbf{B} \hat{z}$. We shall consider only distributions independent of $z$.

An arbitrary-but differentiable-profile, $n_{0}(r)$, accompanied by $\phi_{0}(r)$, where $(1 / r)(d / d r) r(d / d r) \phi_{0}=-4 \pi q n_{0}$, will satisfy the equations of motion. In this steady state, bits of plasma are convected, in circular orbits, with the local " $E \times B$ " velocity. Thus, it is useful to introduce $\omega_{0}(r)$, the local angular velocity,

$$
\omega_{0}(r)=\frac{c}{B_{0}} \frac{1}{r} \frac{d \phi_{0}}{d r}
$$

$\omega_{0}(r)$ is a positive function for a pure-electron plasma, whose potential $\phi_{0}$ is negative, rising to zero at the cylinder edge, and is a negative function for a pure-positron plasma. If we use Poisson's equation, we find

$$
\hat{n}_{0}=\hat{\omega}_{0}+\frac{r}{2} \frac{d \hat{\omega}_{0}}{d r} \quad \text { or } \quad \hat{n}_{0}=\frac{d\left(r^{2} \hat{\omega}_{0}\right)}{d\left(r^{2}\right)},
$$


where $n_{0}(r)=n(0) \hat{n}_{0}(r)$ and $\omega_{0}(r)=\omega(0) \hat{\omega}_{0}(r)$. The density and angular velocities have been scaled by their central values. The magnitude of the central velocity $\omega(0)$ is equal to the "diocotron frequency" $\omega_{p}^{2}(0) / 2 \omega_{0}$ appropriate to the central density. (Here $\omega_{0}$ is the magnitude of the cyclotron frequency; $\omega_{p}$ is the plasma frequency.) Note that for convex profiles, the frequency profile dominates the density profile. For the particular class of convex profiles we will be studying in some detail, we have

$$
\begin{array}{ll}
\hat{n}_{0}(r) & =\left[1-\left(\frac{r}{b}\right)^{2 p}\right], \\
\hat{\omega}_{0}(r) & =\left[1-\frac{1}{1+p}\left(\frac{r}{b}\right)^{2 p}\right],
\end{array}
$$

The frequency at the edge of the plasma has the value $\hat{\omega}_{0}(b)=p /(1+p)$.

Imagine a small perturbation about this steady state. If we write $n(r, \theta, t)=n_{0}(r)+n_{1}(r, \theta, t)$, and a similar expression for $\phi$, we are led to the equations

$$
\frac{\partial n_{1}}{\partial t}+\omega(r) \frac{\partial n_{1}}{\partial \theta}-\gamma(r) \frac{\partial \phi_{1}}{\partial \theta}=0 \quad \text { and } \nabla^{2} \phi_{1}=-4 \pi q n_{1} \text {, }
$$

where $\gamma(r)=\left(c / B_{0}\right)(1 / r)\left(d n_{0} / d r\right)$. These equations are derived under the assumption that $\left|n_{1}(r, \theta, t)\right| \ll n_{0}(r)$ and $\left|\phi_{1}\right| \ll\left|\phi_{0}\right|$. If we represent the angular dependence of density and potential by functions proportional to $\exp (\operatorname{im} \theta)$, we achieve the further simplification,

$$
\begin{aligned}
& \frac{\partial n}{\partial t}(r, t)+i m\left[\omega_{0}(r) n(r, t)-\gamma(r) \phi(r, t)\right]=0, \\
& r \frac{\partial}{\partial r} r \frac{\partial}{\partial r} \phi(r, t)-m^{2} \phi(r, t)=-4 \pi q r^{2} n(r, t) .
\end{aligned}
$$

(The $m$ index has been suppressed.)

\section{A. The experiment-The initial-value problem}

These equations form the basis for our analysis. Let us idealize the experiment by denoting the time at which the driving burst has ceased as $t=0$, and imagining that the $m$ mode has been displaced from steady state by $n(r, 0)$ $=n(0) \delta n(r)$, at that time. The subsequent evolution is described via an initial-value problem, whose analysis calls for Laplace transformation. One then obtains a differential equation for the transformed potential, in which the usual transform parameter $s$ has been written as $s=-i \omega m$. Thus, the conventional half-plane of analyticity-here, $\operatorname{Re}(s)>0$ becomes the upper half-plane $\operatorname{Im}(\omega)>0$, and singular points with $\operatorname{Im}(\omega)<0$ are associated with damping.

The equation,

$$
\begin{aligned}
& {\left[\omega_{0}(r)-\omega\right]\left(r \frac{d}{d r} r \frac{d}{d r}-m^{2}\right) \tilde{\phi}(r, \omega)} \\
& \quad+4 \pi q r^{2} \gamma(r) \tilde{\phi}(r, \omega)=\frac{4 \pi i q r^{2} n(r, 0)}{m},
\end{aligned}
$$

may be simplified, if we use the scaling mentioned earlier, and write $\tilde{\phi}(r, \omega)=4 \pi i q[n(0) / m \omega(0)] \varphi(r, \omega)$. Then,

$$
\left[\omega_{0}(r)-\omega\right] \mathbb{L}_{m} \varphi(r, \omega)+\hat{\gamma}(r) \varphi(r, \omega)=-\delta n(r),
$$

where $\mathbb{L}_{m}$ is the radial part of the Laplacian for the $m$ th mode and $\hat{\gamma}(r)=(2 / r)\left(d \hat{n}_{0} / d r\right)$. With the introduction of the Green's function $g\left(r, r_{0}, \omega\right)$, which obeys

$$
\begin{aligned}
& {\left[\omega_{0}(r)-\omega\right] \mathbb{L}_{m} g\left(r, r_{0}, \omega\right)+\hat{\gamma}(r) g\left(r, r_{0}, \omega\right)} \\
& =-\left[\omega_{0}\left(r_{0}\right)-\omega\right] \delta\left(r-r_{0}\right),
\end{aligned}
$$

the solution becomes

$$
\varphi(r, \omega)=\int_{0}^{a} d r_{0} g\left(r, r_{0}, \omega\right) \frac{\delta n\left(r_{0}\right)}{\left[\omega_{0}\left(r_{0}\right)-\omega\right]}
$$

To get to the point of this study, consider the Green's function for the case in which the plasma fills a perfect, conducting cylinder. Then $b \equiv a$ and the boundary condition is $\varphi(a)=0$. Let $f_{1}$ be the solution to the homogeneous equation regular at the origin, and $f_{2}$ the second solution. Let $f_{3}=\left[f_{2}(a) f_{1}(r)-f_{1}(a) f_{2}(r)\right]$ be the solution vanishing at $r=a$. Then,

$$
g\left(r, r_{0}, \omega\right)=-f_{1}\left(r_{<}\right) f_{3}\left(r_{>}\right) / W\left(f_{1}, f_{3}\right)_{0},
$$

where $r_{<}, r_{>}$denote the smaller/greater of the quantities $r$, $r_{0}$, and $W$ is the Wronskian of the pair of solutions, evaluated at $r_{0}$. Suppose we detect the electric field at the wall, $\mathscr{E}(\omega)=-\varphi^{\prime}(a, \omega)$. After evaluating the Wronskians, we find

$$
\begin{aligned}
\mathscr{E}(\omega) & =\frac{1}{f_{1}(a, \omega)} \int_{0}^{a} d r_{0}\left(\frac{r_{0}}{a}\right) f_{1}\left(r_{0}, \omega\right) \frac{\delta n\left(r_{0}\right)}{\left[\omega_{0}\left(r_{0}\right)-\omega\right]} \\
& =\frac{\mathscr{N}(\omega)}{\mathscr{D}(\omega)},
\end{aligned}
$$

the quantity whose Laplace inversion gives the time dependence of the signal.

The dominant features of the signal are controlled by the singularities in $\mathscr{N}$ and $\mathscr{Q}$. Note that while $\mathscr{N}$ depends upon the form of the perturbation, i.e., upon initial conditions, $\mathscr{Q}$ does not; it is a property of the undisturbed system. Let us restrict our discussion to smooth density profiles that are never increasing, $d n_{0} / d r \leqslant 0$. (There is a slight distinction to be made between $<0$ and $\leqslant 0$, in the analysis. ${ }^{3}$ We neglect it, for it does not affect our particular results.) In this case, $\mathscr{L}(\omega)$ has no zeros. There is no point spectrum. [If we violate the condition by permitting discontinuous behavior in $n_{0}$, we will find a real point spectrum. For example, the profile $\hat{n}_{0}=1,0 \leqslant r<b$, and zero elsewhere, gives $m \omega=m-1$ $+(b / a)^{2 m}$. Further discussion of this case lies ahead.]

One sees easily that there must be a continuous spectrum, the set of all values assumed by $\omega_{0}(r)$. [Merely inspect the integral equation for $n(r, \omega)$.] The continuous spectrum manifests itself in the functional properties of the regular solution, $f_{1}(\omega)$, which is analytic in the complex $\omega$ plane cut along the reals from $\min \left(\omega_{0}\right)$ to $\max \left(\omega_{0}\right)$, or from $\hat{\omega}_{0}(a)$ to 1 . The function $\mathscr{N}(\omega)$, defined as a Cauchy integral, is also analytic in the same cut plane, as long as the perturbation is sufficiently smooth. Such behavior in $\mathscr{N}(\omega)$ and $\mathscr{Z}(\omega)$ permits us to describe the time dependence of the signal in 


\section{Complex $\omega$-Plane}

A

$\mathrm{Br}$

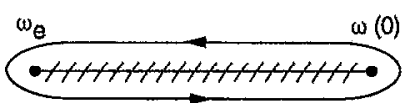

B

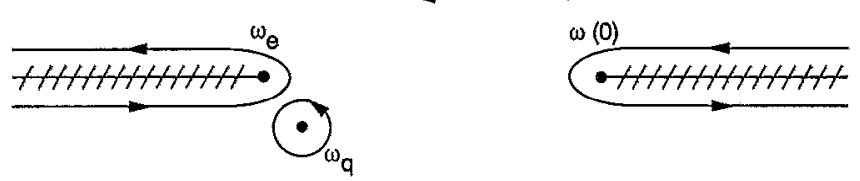

FIG. 1. Two possibilities for the Laplace inversion in the complex $\omega$ plane are shown. In the first, the Bromwich (inversion) contour is deformed about the branch cut; in the second, it is deformed to follow the analytic continuation of the "signal" $\varnothing(\omega)$. In so doing, one collects the contribution of the quasimode pole, $\omega_{q}$

general terms: the integral over a finite range of frequencies giving, possibly, quasiexponential behavior for some intermediate epoch, and oscillatory, but aperiodic behavior at long times. The latter will be governed by the two frequencies, $\min \left(\omega_{0}\right)$ and $\max \left(\omega_{0}\right)$, and by aperiodic multipliers, functions of time whose nature depends on the form of the perturbation as well as the profiles. The appropriate contour for Laplace-inversion, in this case, is shown in Fig. 1.

But the location of the quasimode is independent of initial conditions! It is associated with the zeros of $\mathscr{Z}(\omega)$ or, more precisely, with zeros of the analytic continuation of $f_{1}(a, \omega)$ across the cut. We can isolate the quasimode, as well as the long-time behavior, by using an alternate contour, also shown in Fig. 1. When the real part of the complex zero lies between $\min \left(\omega_{0}\right)$ and $\max \left(\omega_{0}\right)$, it determines a "resonant radius," through the equation $\operatorname{Re}(\omega)=\omega_{0}(r)$. Here it is that the connection with Landau damping appears. There is a shape, $f_{1}^{*}(r, \omega)$, associated with the quasimode, but that function, being generated through analytic continuation in the complex $r$ plane, is generally discontinuous at the resonant radius, and is not a proper mode. ${ }^{3}$

The remainder of this paper will be devoted to the study of the regular solution, $f_{1}$.

\section{B. General features of the homogeneous equation}

This section is somewhat technical, and may be omitted by the reader interested primarily in numerical results.

A change of variables facilitates the general discussion, when the frequency profile is nonincreasing. Define $z$ and $p(z)$ through

$$
(1-\omega) z=1-\hat{\omega}_{0}(r), \quad p(z)=\frac{1}{2} \frac{d(\ln z)}{d(\ln r)},
$$

and consider the general case, in which $\omega$ and $z$ are complex. The interval $0 \leqslant r<b$ becomes a ray emanating from $z=0$. In the case of power-law profiles, for example,

$$
(1-\omega) z=\frac{1}{1+p}\left(\frac{r}{b}\right)^{2 p}, \quad p(z)=p,
$$

so that $p(z)$ may be regarded as the "local power." Generally, then

$$
\begin{aligned}
& p(z)=\frac{1}{2} \frac{r}{1-\hat{\omega}_{0}(r)}\left(-\frac{d \hat{\omega}_{0}(r)}{d r}\right)=\frac{\hat{\omega}_{0}(r)-\hat{n}_{0}(r)}{1-\hat{\omega}_{0}(r)}, \\
& (1-\omega) z p(z)=\frac{1}{2} r\left(-\frac{d \hat{\omega}_{0}(r)}{d r}\right)=\hat{\omega}_{0}(r)-\hat{n}_{0}(r),
\end{aligned}
$$

and

$$
[z p(z)]_{z}+1=\left(\frac{d \hat{n}_{0}}{d r}\right) /\left(\frac{d \hat{\omega}_{0}}{d r}\right) .
$$

With these new quantities, the equation for $\psi$, defined through $\varphi=r^{m} \psi$, becomes

$$
\frac{d^{2} \psi}{d z^{2}}+\left(\frac{[z p(z)]_{z}+m}{z p(z)}\right) \frac{d \psi}{d z}+\left(\frac{[z p(z)]_{z}+1}{z p(z)}\right) \frac{\psi}{1-z}=0,
$$

and the boundary condition is imposed at "the edge,"

$$
z=z_{e}=\frac{\left(1-\omega_{e}\right)}{(1-\omega)},
$$

where we denote the frequency at the edge of the plasma, $\hat{\omega}_{0}(b)$, by $\omega_{e}$. Note that when $m=1$, the equation has the obvious solution $\psi=A(1-z)$. In this special case, $\omega=\omega_{e}$ and the boundary condition is imposed at $z=1$. Generally, $z_{e}$ will be close to, but distinct from, the singular point, $z=1$. Note, too that when $p(z)=p$, our equation becomes the hypergeometric differential equation,

$$
\frac{d^{2} \psi}{d z^{2}}+\left(\frac{p+m}{p}\right) \frac{1}{z} \frac{d \psi}{d z}+\left(\frac{p+1}{p}\right) \frac{\psi}{z(1-z)}=0 .
$$

Let us inquire about the nature of the solutions, $\psi$. Begin with Eq. (13), which has regular singular points at $z=0$ and $z=1$. The solution we desire, regular at the origin, is the hypergeometric function $F(\bar{a}, \bar{b} ; \bar{c} ; z)$, with $\bar{c}=\bar{a}+\bar{b}+1$ $=(p+m) / p$ and $-\bar{a} \bar{b}=(p+1) / p$. It is required to satisfy a boundary condition at $r=b$, or at

$$
z=z_{e}=\frac{\left(1-\omega_{e}\right)}{(1-\omega)}=\frac{1}{(1-\omega)(1+p)} .
$$

Thus, the important function $f_{1}(\omega)$, introduced earlier, is $b^{m} F\left[\vec{a}, \bar{b} ; \bar{c} ; z_{e}(\omega)\right]$, which is known to be multivalued. Indeed, the hypergeometric function is analytic in the complex plane cut along the reals from $z=1$ to $\infty$. Then, the corresponding $f_{1}$ is seen to be analytic in the $\omega$ plane cut from $p /(1+p)$ to 1 , that is, from $\min (\omega)$ to $\max (\omega)$, as we conjectured earlier.

The boundary condition produces an eigenvalue equation, from which we deduce $z_{e}$, then $\omega$. For example, case 1 gives $z_{e}=z_{*}$, the latter quantity being one of the zeros of the hypergeometric function. We have found that, in all cases, $z_{e} \approx 1$, or $\omega \approx \omega_{e}$. This is a crucial result of our investigation; it bears out our "hunch" about a nearby singularity, or "quasimode." Henceforth, we shall be particularly interested in $\psi$ near $z=1$, which appears to be a singular point of the 
differential equation. In that neighborhood, with $\xi=1-z$, the usual analysis shows the general solution to be a combination,

$$
\psi(\xi)=a_{2} \psi_{2}(\xi)+a_{3} \psi_{3}(\xi),
$$

where $\psi_{2}(\xi)$ is regular, and $\psi_{3}(\xi)=\lambda(\xi) \xi \ln \xi$, with $\lambda(\xi)$ regular and $\lambda(0) \neq 0$. Our knowledge of $F$ gives us the values of $a_{2}$ and $a_{3}$ and, as we discuss later, the first few terms in the series expansion of $\psi_{2}$ and $\psi_{3}$ will give $z_{e}$ and therefore $\omega$, accurately.

Turn now to the general equation, Eq. (12). We should like to know how typical the results for power-law profiles might be. Suppose that $p(z)$ is regular in a region of the $z$ plane, containing the points $z=0$ and $z=1$. [For power-law profiles, $p(z)$ is simply constant.] Further, we must require that $p(z)$ (i.e., the slope of the frequency profile) never vanish in the region. Then, the point $z=0$ is a regular singular point, of the same nature as its counterpart in Eq. (13). Again, there is a regular solution, and, as long as $p(z)$ is regular, we can continue the solution into a neighborhood containing the point $z=1$, where we seek eigenvalues. If we inspect the differential equation, we see that the form of the solution about $z=1$ is then linked to the behavior of $[z p(z)]_{z}+1$, the ratio of the density slope to the frequency slope. Should that quantity vanish at $z=1$, the singularity will, generally, be removed and the nature of the solution altered. (Should the slope of the frequency profile vanish, the singularity would be aggravated. But we have shut out that possibility.) The point $z=1$, close to $z_{e}$, corresponds to (complex) $r$ close to $r=b$. If we choose an equilibrium density profile whose slope at the edge is quite distinct from zero, as in the power-law profile, we may expect $z=1$ to remain a regular singular point. If, however, we deliberately choose, say, a polynomial profile with slope vanishing at $r=b,\left(z=z_{e}\right)$ or a density-profile decreasing exponentially toward a distant wall, matters would be delicate, $[z p(z)]_{z}+1$ being very small at $z=1$, vanishing there as $\omega$ approaches the edge frequency (and $z_{e}$ approaches 1 ). Then, the point $z=1$ becomes a regular point. The matter of vanishing or nonvanishing slope is important when we ask about the effect of a gap, an effect we discuss later. We now turn to details of the particular model-power-law profiles.

\section{Power-law profiles}

With $\varphi=r^{m} \psi$, and $\psi$ proportional to $F$, we study hypergeometric functions when $\bar{c}=1+\bar{a}+\bar{b}$. For us, $\bar{a}, \bar{b}=\beta \overline{+} \gamma$, where $\beta=m / 2 p$ and $\gamma={ } \beta^{2}+(1+p) / p$. Thus $\bar{b}>0$ and $\bar{a}<0$. Graphical analysis $(m>1)$ gives $-1<\bar{a}<0$ and $\bar{b}>1, \bar{c}-\bar{b}>0$, and $\bar{c}>1$. In the special case, $m=1$, we have $\bar{a}=-1$, and $\bar{b}=\bar{c}, \bar{b}=(1+p) / p$. The hypergeometric function may be defined by the series

$F(\bar{a}, \bar{b} ; \bar{c} ; z)=\sum \frac{(\bar{a})_{n}(\bar{b})_{n}}{(\bar{c})_{n}} \frac{z^{n}}{n !}$,

which converges for $|z|<1$,

and its continuation, analytic in the $z$-plane cut along the real axis from $z=1$ to $\infty$. Our function may be continued via the representation

$$
F(\bar{a}, \bar{b} ; \bar{c} ; z)=\frac{\Gamma(\bar{c})}{\Gamma(\bar{b}) \Gamma(\bar{c}-\bar{b})} \int_{1}^{\infty} \frac{d u}{u^{1+b}} \frac{(u-1)^{\bar{a}}}{(u-z)^{\bar{a}}},
$$

the phases chosen for the fundamental branch so that $F$ is real on the real axis, $\operatorname{Re}(z)<1$. With our choice of $(\bar{a}, \bar{b}, \bar{c})$, the prefactor cannot vanish, and the integrand is positive for real $z<1$. That is, there are no real zeros in that interval, and, therefore, no eigenvalues, no real frequencies, and no normal modes in case 1 . If we study, instead, the analytic continuation, $\stackrel{*}{F}(\overline{\mathrm{a}}, \overline{\mathrm{b}} ; \overline{\mathrm{c}} ; \mathrm{z})$, we will find complex zeros, $z_{*}$, and associated quasimodes. The associated mode shapes are discontinuous, being constructed from $F$ when $r$ is less than the resonant radius, and $\stackrel{*}{F}$ when $r$ is greater.

\section{Zeros of the continued hypergeometric function}

Let us denote the zeros, generally, by $z_{*}$. We are particularly interested in $z_{*} \approx 1$. To these zeros will correspond frequencies $\omega$ near $\omega_{e}$. We shall find $z_{*}=1+\left(\epsilon_{1}-i \epsilon_{2}\right)$, the $\epsilon$ 's small and positive. Then, since the eigenvalue condition is $z_{e}=z_{*}$,

$$
\omega \approx \frac{p}{1+p}\left[\left(1+\frac{\epsilon_{1}}{p}\right)-i \frac{\epsilon_{2}}{p}\right]=\omega_{e}\left[\left(1+\frac{\epsilon_{1}}{p}\right)-i \frac{\epsilon_{2}}{p}\right],
$$

and the frequency of the mode lies inside the frequency profile, $\hat{\omega}_{0}(r)$.

We may evaluate $F$ on nearby sheets by continuing the phases associated with factors appearing in the integral. A coarse numerical survey of the second sheet uncovers an isolated zero close to the branch point, $z=1$, no other zero remotely close. Thus, the characteristic "signature" of the mode should be detected in an experiment like the one carried out by Pillai and Gould. (The signature would be absent only if an unusual initial distribution were orthogonal to the mode-if it were excited barely.) The convenient location of the zero simplifies the calculation to a point where it may be done with a pocket calculator for, being interested only in the neighborhood of $z=1$, we need only a few terms of an appropriate series expansion of the hypergeometric function. From the literature, then, with $\xi=1-z$, and $\psi(z)$ the digamma function,

$$
\begin{aligned}
& F(\bar{a}, \bar{b} ; \bar{a}+\bar{b}+1 ; z) \\
& =\frac{\Gamma(\bar{a}+\bar{b}+1)}{\Gamma(\bar{a}+1) \Gamma(\bar{b}+1)}\left(1+\bar{a} \bar{b} \xi \sum_{0}^{\infty} \frac{(\bar{a}+1)_{n}(\bar{b}+1)_{n}}{n !(n+1) !}\right. \\
& \left.\quad \times\left(\ln \xi-B_{n}\right) \xi^{n}\right),
\end{aligned}
$$

with $\quad B_{n} \equiv \ln \mathscr{P}_{n}=\psi(n+1)+\psi(n+2)-\psi(\bar{a}+n+1)$ $-\psi(\bar{b}+n+1)$. If $|\arg (\xi)|<\pi$, we have the fundamental branch. Analytic continuation is achieved by continuing the argument of the logarithm. If we add $2 \pi i$ to the logarithm, and note that $(2)_{n}=(n+1)$ !, we find 


$$
\begin{aligned}
& \text { W }(\bar{a}, \bar{b} ; \bar{a}+\bar{b}+1 ; z) \\
&= F(\bar{a}, \bar{b} ; \bar{a}+\bar{b}+1 ; z)+2 \pi i \frac{\Gamma(\bar{a}+\bar{b}+1)}{\Gamma(\bar{a}) \Gamma(\bar{b})} \\
& \times(1-z) F(\bar{a}+1, \bar{b}+1 ; 2 ; 1-z),
\end{aligned}
$$

for the neighboring branch. In practice, however, a few terms of the series suffice to locate the zero quite well. In fact, the first two terms are effective, and deserve consideration. They are

$$
\begin{aligned}
F(\bar{a}, \bar{b} ; \bar{a}+\bar{b}+1 ; z) \approx & \operatorname{const}\{1+\bar{a} \bar{b} \xi[\ln \xi+\psi(\bar{a}+1) \\
& +\psi(\bar{b}+1)-2 \psi(1)-1]\},
\end{aligned}
$$

with an error $O\left(\xi^{2} \ln \xi\right)$. Then, with $\not A=1+1 / p=-\bar{a} \bar{b}$, $B_{0} \equiv B$, we may write the equation for the zeros as

$$
\xi_{1} \ln \xi_{1}=\epsilon,
$$

where $\epsilon=1 / \mathscr{H} \mathscr{B}$ is a dimensionless parameter and $\xi_{1}=\xi / \mathscr{R}$. The equation can be solved in many ways; the most simple is an iterative scheme,

$$
\left(\xi_{1}\right)_{n+1}=\frac{\epsilon}{\left(\operatorname{In} \xi_{1}\right)_{n}},
$$

which requires complex arithmetic. Or, one may manipulate real and imaginary parts to obtain the pair of equations,

$$
\exp \left(-\frac{\Theta}{\tan \Theta}\right)=-\epsilon \frac{\sin \Theta}{\Theta}=\left|\xi_{1}\right|, \quad \Theta=\arg \xi_{1},
$$

which are to be solved in $2 \pi>\Theta \geqslant \pi$. Thus, from the pair $(m, p)$ we calculate $\epsilon$, then $\Theta$, then $\xi$. For example, in the regime in which $\epsilon$ is small, one finds

$$
\left|\xi_{1}\right|=\frac{\epsilon}{\ln (1 / \epsilon)}+\cdots, \quad \Theta=\pi\left(1+\frac{1}{\ln (1 / \epsilon)}+\cdots\right),
$$

so that-to leading order-

$$
z_{*} \approx 1+\frac{p}{1+p} \frac{1}{\ln (1 / \epsilon)}\left(1-i \pi \frac{1}{\ln (1 / \epsilon)}\right) .
$$

But there is a nice general result: The relation between $\xi$ and $\Theta$ is independent of $m$. It follows that, in case 1 , the complex frequency of the quasimode lies on the curve

$$
\omega=1-\frac{1}{1+p\left[1+(\sin \Theta / \Theta) e^{i \Theta}\right]}, \quad \pi \leqslant \Theta<2 \pi,
$$

particular values of $\Theta$ depending on $m$ and $p$. The curve is displayed as Fig. 2.

Several comments: First, the general shape of the curve perseveres as we go to approximations of higher order. Figure 3 , based on a converged, many-term representation of $F$ shows this, clearly. Second, the "unphysical" portion, in which $\operatorname{Re}(\omega)<\omega_{e}$ and "there is nothing to resonate with" is not an artifact. For those $(m, p)$, the full, time-dependent solution shows no quasiexponential behavior. Finally, before becoming alarmed by the peculiar, egg-shaped, curve one should recall that $\operatorname{Re}(\omega)$ is the wave speed, the true frequency divided by $m$, the mode number, and $\operatorname{Im}(\omega)$ is the damping coefficient divided by $m$. The latter quantities, displayed in Figs. 4 and 5 behave in a more familiar manner.

\section{Locus of Complex Frequencles, Profile $(p=4)$, No Gap}

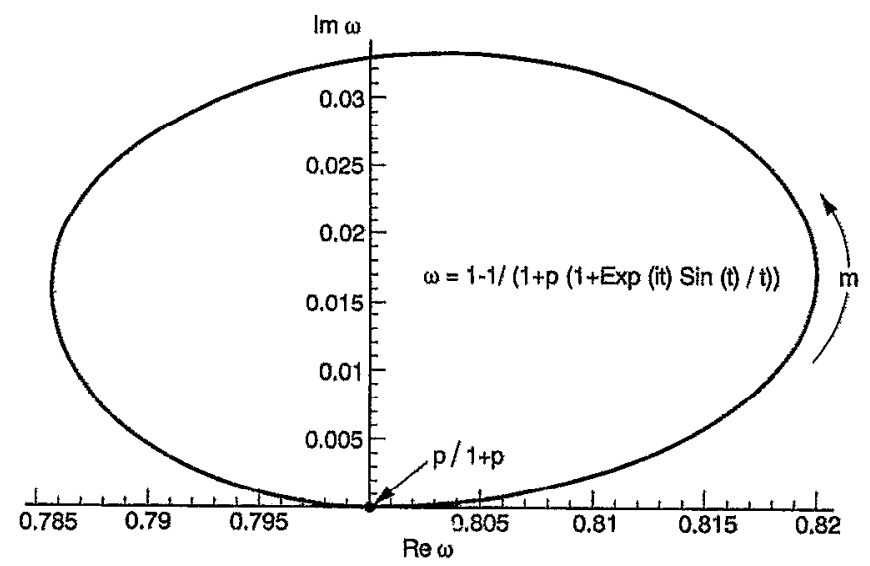

FIG. 2. In the simplest approximation to the eigenvalue equation for gapless systems, complex quasimode frequencies must lie on the simple curve described by Eq. (17), for $p=4$. Particular mode numbers $m$ correspond to particular points on the curve. Remember that these are true complex fre-

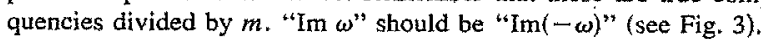

We can estimate the behavior of the modes in the two limits $(m-1) / p \rightarrow 0$ and $\infty,(\epsilon \rightarrow 0$ and $\infty ; \Theta \rightarrow \pi$ and $2 \pi)$ from Eq. (17). The lower limit is controlled by $\psi(\bar{a}+1)$. If $\bar{a}$ lies close to -1 , the digamma function is very large and negative, $B$ large and positive. The upper limit is controlled by $\psi(\bar{b}+1)$ and $B$ is large and negative. We find, in leading order.

\section{Complex Frequency vs Mode, Protile $(p=4)$, No Gap}

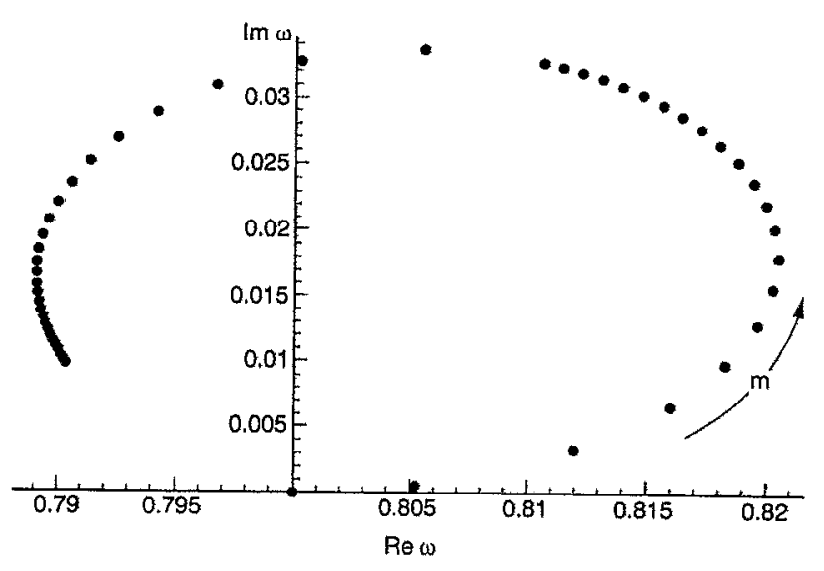

FIG. 3. Complex frequencies for different $m$ values beginning with $m=1$, for the full, converged eigenvalue equation. The $m$ intervals are usually unity. The profile is $p=4$; there is no gap. "Im $\omega$ " should be " $\operatorname{Im}(-\omega)$ " (compare with Fig. 2). 
Frequency vs Mode, Quadratic Proflle, No Gap

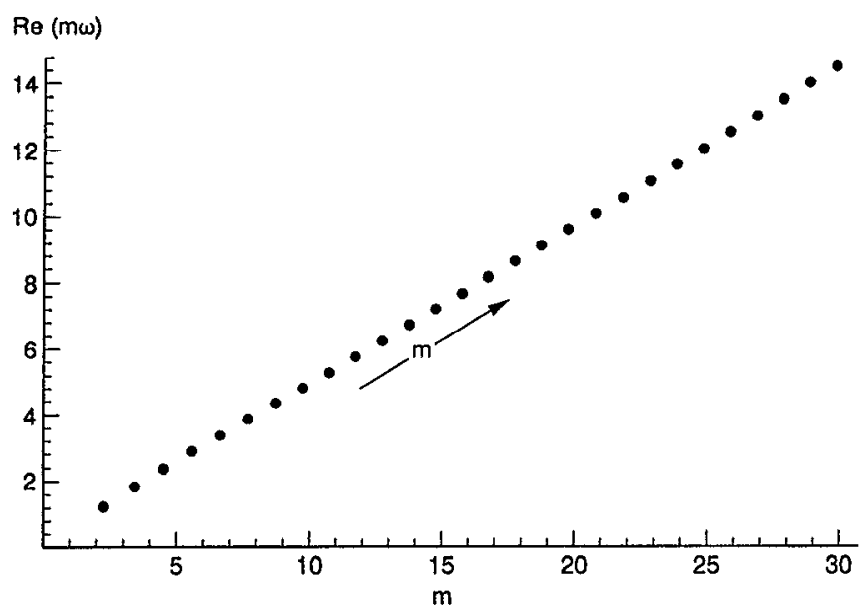

FIG. 4. The real part of the true complex frequency versus the mode number.

$$
\begin{aligned}
\frac{\omega}{\omega_{e}}= & 1+\frac{1}{1+p}\left(\frac{m-1}{2 p+1}\right)+i \pi \frac{1}{1+p}\left(\frac{m-1}{2 p+1}\right)^{2} \\
& +\cdots \frac{m-1}{p} \rightarrow 0, \\
= & 1-\frac{1}{1+p}\left(\frac{1}{\ln (m / p)}\right)+i 2 \pi \frac{1}{1+p}\left(\frac{1}{\ln (m / p)}\right)^{2} \\
& +\cdots \frac{m-1}{p} \rightarrow \infty,
\end{aligned}
$$

results borne out by a more precise computation. Thus, for large $m / p$, the waves are dispersionless; the true damping increases with $m$, almost linearly. For very flat profiles and moderate $m$, so that $(m-1) / p \rightarrow 0$, the modes cluster at the

\section{Damping vs Mode, Quadratic Profile, No Gap}

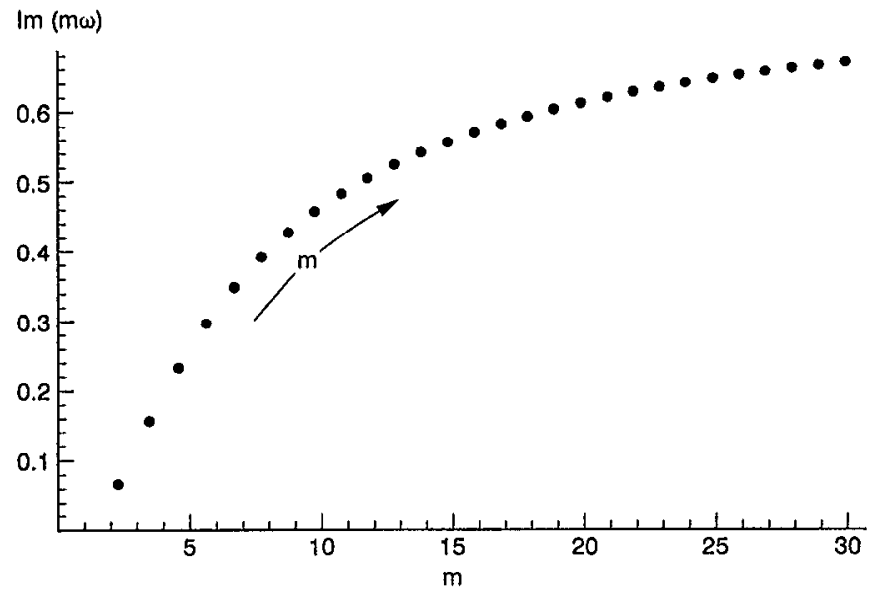

FIG. 5. Imaginary part (damping) of the true complex frequency versus the mode number. "Im $m \omega$ " should be "Im $(-m \omega)$." edge frequency. Indeed, $\omega_{e}$ is the limit point for the wave speed (our $\omega$, the true frequency divided by $m$ ) in both limiting cases.

\section{Boundary conditions}

In the three cases we study, the boundary condition at the edge of the plasma is homogeneous of the form

$$
\Phi(\omega, \lambda) \equiv\left(\tilde{\phi}(r, \omega)+\lambda \frac{d \tilde{\phi}}{d r}(r, \omega)\right)_{r=b}=0 .
$$

So far, our discussion has been limited to the first case, in which the plasma fills a perfectly conducting cylinder. Then, $b=a$, and the component of electric field parallel to the surface of the shell must vanish. We infer that $\tilde{\phi}(b, \omega)=0$, or $\lambda=0$. In case 2 , where again $b=a$, we find that $\lambda / a$, which depends both upon the conductivity of-say, copper, and the frequency of the mode, is quite small. Also, $\lambda$ is itself a function of $\omega$. Here $\lambda(\omega)$ has the familiar "skin-depth" form $^{3,4}$

$$
\lambda=-i\left(\frac{m \omega}{c}\right)\left(\frac{a}{m}\right)^{2} \sqrt{\frac{m \omega}{4 \pi \sigma}} \exp \left(-i \frac{\pi}{4}\right) .
$$

In the third case, boundary conditions at the vacuum gap yield

$$
\lambda=a \frac{\Theta}{m} \frac{\left(1-\Theta^{2 m}\right)}{\left(1+\Theta^{2 m}\right)}, \quad \Theta=\frac{b}{a} .
$$

Thus $\lambda / a$ is small when the gap is small, and when it is large. (For most $m, \lambda / a<0.25$.) A useful approximation then, for cases 2 and 3 , is

$\Phi(\omega, \lambda) \equiv\left(\tilde{\phi}(r, \omega)+\lambda \frac{d \tilde{\phi}}{d r}(r, \omega)\right)_{r=b} \approx \tilde{\phi}(b+\lambda, \omega)=0$,

reducing these cases to case 1 . We shall use this form ("extrapolated boundary") in treating the imperfect conductor and, safely, when the gap is small $(\lambda \ll b)$.

In case 2 , then, we expect only a small shift in frequency, controlled by the small quantity $\sqrt{(\omega / \sigma)}$. Thus, we use the unshifted frequency in the computation of $\lambda$, and obtain for the frequency shift $\delta \omega$,

$$
\begin{aligned}
\frac{\delta \omega}{\hat{\omega}_{0}(b)} & \approx \frac{2}{z_{*}} \frac{\omega a}{c} \sqrt{\frac{\omega}{4 \pi m \sigma}} \exp \left(i \frac{\pi}{4}\right) \\
& \approx \frac{\omega a}{c} \sqrt{\frac{\omega}{2 \pi m \sigma}}(1+i) .
\end{aligned}
$$

The effect of finite conductivity is to decrease the damping, to destabilize the mode. In the language of electrophysics, these "waves," $\exp [i m(\theta-\omega t)]$, are "negative energy waves." [All frequencies, and $\sigma$, are in units of $\omega(0)$, the diocotron frequency. And recall that the true frequency is $m \omega$, and that a negative imaginary part corresponds to damping.] The expression for the shift may be brought closer to experiment by noting that $R$, the resistance associated with the eddy currents flowing in the wall, is given by $R=(1 / c)$ $\times(\sqrt{ } 2 \pi \omega / \sigma)$. Thus, the decrease in damping is proportional 
Complex Frequency vs Profile, $m=2$, No Gap

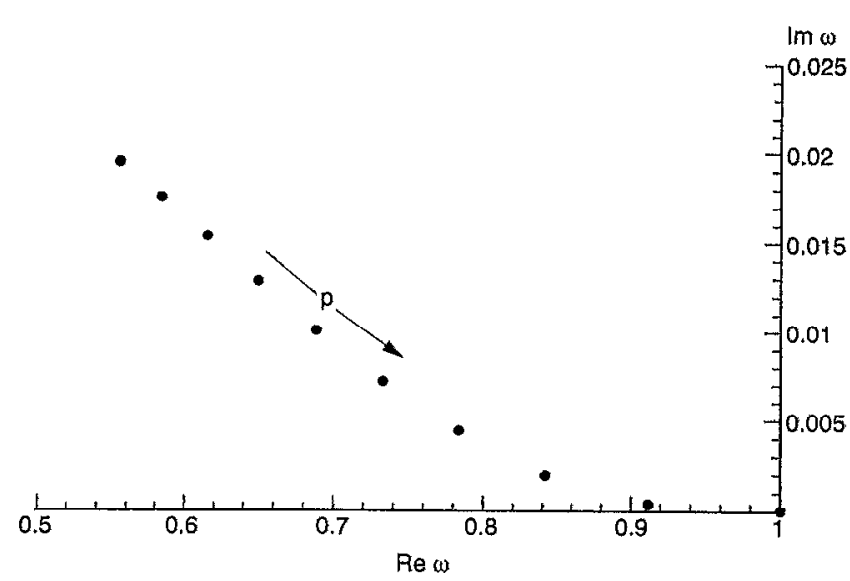

FIG. 6. Dependence of the complex frequency upon mode shape. The limit. as $p \rightarrow \infty$, (uniform, filling distribution) is $\omega \rightarrow 1$. "Im $\omega$ " should be "Im(- $-\omega)$,"

to the resistance. White, Malmberg, and Driscoll, ${ }^{5}$ and Pillai and Gould ' have observed such an effect by loading the containing cylinder with lumped resistances. The change in damping is linear in $R$ over a considerable range.

A final observation about case $2: \lambda$ is complex, and the proof $^{3}$ of the empty point spectrum is spoiled thereby. We will not investigate the point spectrum: rather, since $\lambda / a \leqslant 1$, we regard the wall resistance as causing a small perturbation of the quasimode found for the ideal conductor.

\section{The gap}

In case 3, a vacuum gap separates the plasma from the wall. We change from $\tilde{\phi}=r^{m} \psi$ to $\psi$, the boundary condition becoming

$$
\left(\psi+\lambda^{\prime} z \frac{d \psi}{d z}\right)_{z=z_{e}}=0, \quad \lambda^{\prime}=\frac{p}{m}\left(1-\Theta^{2 m}\right),
$$

a more complicated expression involving hypergeometric functions. When the gap is small, we may use the "extrapolated boundary" condition to estimate the frequency shift,

$$
\frac{\delta \omega}{\omega_{e}} \approx-\frac{2}{m} \frac{\left(1-\Theta^{2 m}\right)}{\left(1+\Theta^{2 m}\right)} .
$$

Thus, a small gap diminishes the real part of the frequency, but does not affect the damping. For larger, more realistic gaps-and note how quickly $\lambda^{\prime}$ becomes $p / m$, or "wall at infinity"-we must use the correct boundary condition. The condition may be used in conjunction with the simple approximation to the hypergeometric function noted earlier, or with a representation involving many terms. The first approach leads to a more complicated version of Eq. (16),

$$
\begin{gathered}
\xi_{1}\left[\lambda^{\prime}+\left(1+\lambda^{\prime}\right) \ln \xi_{1}\right]=\epsilon\left[\left(1+\lambda^{\prime \prime}\right)+\lambda^{\prime \prime} \ln \xi_{1}\right], \\
\lambda^{\prime \prime}=\lambda_{\lambda}^{\prime},
\end{gathered}
$$

an equation adequate to determine mode frequencies for profiles that are not too flat. Specifically, it has no solutions

\section{Complex Frequency vs Gap, Quadratic Profile, $m=2$}

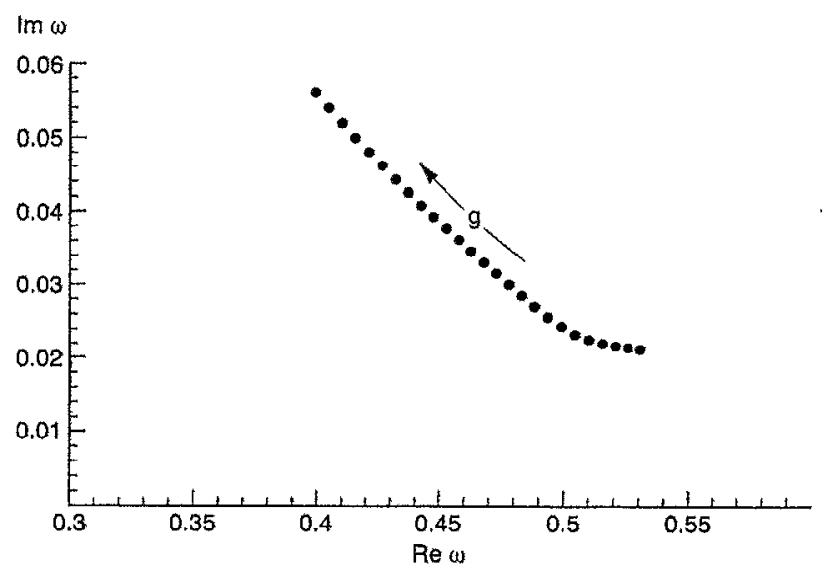

FIG. 7. Dependence of the complex frequency upon gap size. As noted in the text, small gaps produce little change in damping. "Im $\omega$ " should be "Im( $(-\omega) . "$

$\xi_{1} \approx 0$. This defect, which we discuss later, is not lied to the simple approximation. Rather, it characterizes the power-law profiles. The scheme using many terms requires a computer program. Its results may be seen in Figs. 7 and 8. Figure 8, in particular, shows no sign of the defect.

The limiting case of near-flat profiles, $p \rightarrow \infty$ (nearuniform "patches" of density, or, in the fluid analog, vorticity) is of considerable interest. ${ }^{6}$ In our model, the key parameters approach limits $\bar{a} \rightarrow-1, \bar{b} \rightarrow 1$, and $z_{e} \rightarrow 1$. We have not been able to extract a useful expression for the damping in that limit. But there is a related situation-in which damping is absent-that may be analyzed easily. Suppose that the density profile does not vanish, but falls discontinuously to zero at $r=b$. An example might be

\section{Frequency vs Gap Size, Near-Step Profiles, $p=10,20(m=2)$}

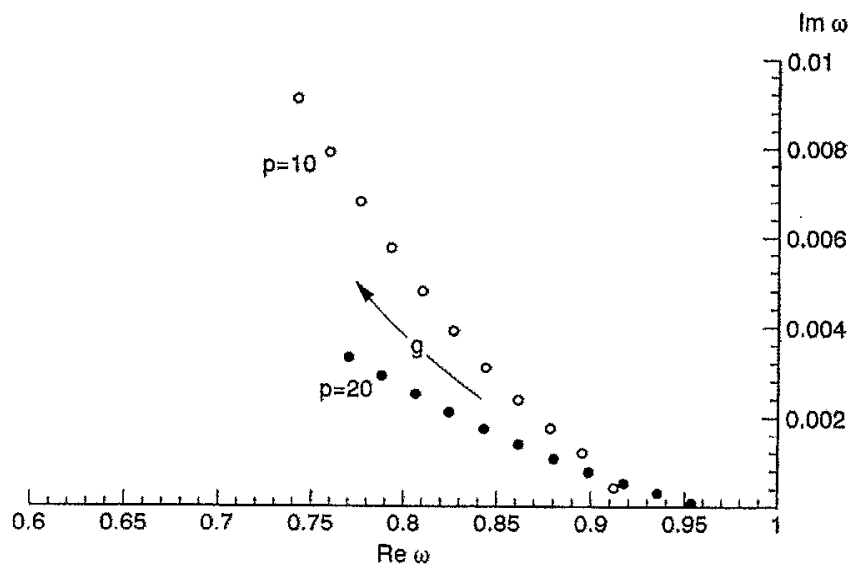

FIG. 8. Dependence of the frequency upon the "flatness" of profile, in systems with gaps. Note vanishing damping as one approaches the "tophat" profile. One can infer from these curves either the approach to teal frequencies as $p-\infty$, gap fixed, or the $g$ dependence when $p \gg 1$, "Im $\omega$ " should be "Im $(-\omega)$." 


$$
\begin{aligned}
\hat{n}_{0}(r) & =\left[1-\left(\frac{r}{c}\right)^{2 p}\right], \quad 0 \leqslant r \leqslant b<c, \\
& =0, \quad b<r \leqslant a .
\end{aligned}
$$

The "top-hat" profile, in which $\hat{n}_{0}$ assumes values 1 and zero, is a limiting case $(c \rightarrow \infty)$. Generally, a convex, discontinuous profile will produce real eigenvalues; the corresponding frequencies may be obtained from the equation

$$
\omega=\hat{\omega}_{0}(b)-(2 / b) \hat{n}_{0}(b) g(b, b, \omega),
$$

the Green's function being the one encountered earlier, with $\hat{\mathcal{X}}(r)=(2 / r)\left(d \hat{n}_{0} / d r\right) H_{c}(r-b)$. (Here $H_{c}=1-H$ is the complement of the Heaviside function.) In the case we will consider that there is only one frequency per $m$ mode. The corresponding eigenfunction is

$$
\begin{aligned}
n(r)= & \frac{1}{4 \pi q} \frac{\varphi(b)}{g(b, b)}(\delta(r-b) \\
& \left.+\frac{H_{c}(r-b)}{\omega-\omega(r)} \frac{2}{r} \frac{d \hat{n}_{0}}{d r} g(r, b)\right) .
\end{aligned}
$$

It displays clearly the "surface-wave" nature of the disturbance. On the other hand, when the density profile is continuous, the disturbance is concentrated in the interior of the plasma, at some "resonant radius."

It is easy to find the frequency when the profile is curved, slightly. Then, the dispersion relation yields

$$
\omega=\hat{\omega}_{0}(b)-\frac{1}{m}\left[1-\left(\frac{b}{a}\right)^{2 m}\right]\left(1-\frac{m}{m+p} \epsilon\right)+\cdots,
$$

with smallness parameter $\epsilon=(b / c)^{2 p}$. Here $\hat{\omega}_{0}(b)$ $=1-\epsilon /(1+p)$ is the edge frequency. [Note the $m=1$ limit. Further, the $p \rightarrow \infty$ limit of Eq. (23) is the $\epsilon=0$ limit, here.] Thus. curvature pushes the mode frequency toward the edge of the continuous frequency band. The reader will note that as we go to the limit of continuous profile, $\hat{n}_{0}(b) \rightarrow 0$, or vanishing gap, $b \rightarrow a$, the edge frequency appears to be a limit point for $\omega$. But no real frequencies exist for these profiles. Clearly, the limiting behavior is delicate; we do not choose to pursue the point, here.

\section{RESULTS AND SUMMARY}

The results of our study might fill a three-dimensional space, described by parameters $(m, p, g)$-mode, profile, and gap. We present a thin sample; the reader is invited to fill in the lacunae.

First, the quasifrequencies that emerge are all "reasonable" when compared with experiment ${ }^{1,2}$ or with other computations. A typical result, for $m=2$, a quadratic profile ( $p$ $=1)$, and the "egg" of Fig. 2 is $\omega=(0.47,0.023)$, which gives a true frequency of $(0.94,0.046) \omega(0)$. Flatter profiles will produce less damping, larger " $Q$ 's." Then, when we study the $m$ dependence, we are struck by the lack of dispersion, as displayed in Fig. 4. Were it not for the modedependent damping, one would expect an initial disturbance containing many angular modes to evolve into a characteristic "shape," which would endure for a perceptible interval of time. Since the damping [Fig. 5] is mode dependent, though small, the shape would be altered slowly, its smaller features disappearing more rapidly. The estimates in Eq. (18) prove to be quite reasonable; more precise numerical calculations appear to show the logarithmic drifts that appear in (18).

Since flat, or "top-hat" profiles produce real modes, the study of the profile dependence of quasimodes contains little that is surprising (see Figs. 6 and 7). As $p$, and therefore "flatness" increases, so damping-which, ultimately, is phase mixing-decreases. For a given profile, creating a gap or, equivalently, compressing the plasma, lowers the frequency of the quasimode. Small gaps do not affect the damping, while large gaps cause it to increase. It appears that the larger the density-or frequency gradient in the profile, the larger is the phase mixing (damping). (That the damping is proportional to the gradient of the density at the resonant radius appears in the perturbation calculations of BDL.)

The last figure, Fig. 8, displays the approach to "tophat" behavior in some detail. One may note both the vanishing of the damping as $p \rightarrow \infty$ for systems containing a gap and the variation of the quasimode with gap size in nearly flat systems.

Finally, one returns to the question: Is the power-law profile, with its discontinuous slope of density and frequency, untypical, too special to provide insights? We have noted that the profile with discontinuous density misses the quasimode effect entirely. How flawed are the predictions that proceed from power-law profiles?

Of course, measured profiles are not so simple. Unfortunately, we do not yet have enough data to test the sensitivity of quasimode frequencies to details of the profiles. For example, Eq. (14) gives the quasimode frequency in terms of edge frequency, mode number, and profile as

$$
\omega=1-\frac{1-\omega_{e}}{z_{*}(m, p)},
$$

$z_{*}$ being the zero of the hypergeometric function. Perhaps, given a complicated but convex profile, one will find that a suitably averaged " $p$ " gives good agreement.

We can imagine one situation in which a power-law model is misleading. Recall Eq. (12). Suppose that a series of experiments, imagined or real, causes the quasimode to approach $\omega_{e}$. In terms of the $z$ variable, $z_{e} \rightarrow 1$. Now, as we have remarked, the behavior of solutions in this $z$ neighborhood depends critically upon

$$
[z p(z)]_{z}+1=\left(\frac{d \hat{n}_{0}}{d r}\right) /\left(\frac{d \hat{\omega}_{0}}{d r}\right) .
$$

If this quantity vanishes at the plasma edge, $z_{e}$, and we drive $z_{e} \rightarrow 1$, the point $z=1$ will be, in the limit, a regular point. Such behavior will occur if the slope of the density vanishes at the edge. If, on the other hand, the slope does not vanish, $z=1$ remains a singular point and the logarithmic behavior of the general solution in its neighborhood would make it impossible to satisfy a general boundary condition like Eq. (19). Then $\omega$ might not be permitted to approach $\omega_{e}$, and the power-law model would prove inadequate to describe the experiment. We have noticed this defect, which seems not to 
be troublesome, in practice. Otherwise, we believe the analysis we have presented in this paper to be more than suggestive, when one deals with real situations.

Note added in proof: We have been directed to a paper ${ }^{7}$ concerned with the motion of vortex filaments in fluid dynamics, in which the authors, E. G. Broadbent and D. W. Moore, deal with equations similar to those in our manuscript. Their analysis proceeds differently, but they do comment about the mathematical difficulty/delicacy of the approach to the "top-hat" profile.

\section{ACKNOWLEDGMENTS}

This work has benefited greatly from valuable discussions with my colleagues Roy Gould and Sateesh Pillai. A portion of the manuscript was written while the author was in residence at The Aspen Center for Physics. He is grateful to the Directors of the Center for providing a stimulating and hospitable environment for research.

\section{APPENDIX: A FORM OF THE HOMOGENEOUS EQUATION SUITABLE FOR MODELING}

If we scale all frequencies with $\omega(0)$ and recall the definition of $\gamma(r)$, the form of the differential equation becomes particularly simple. In fact, with the notation $D f=r(d / d r) f$ and $\Omega(r)=\omega-\hat{\omega}_{0}(r)$, we find

$$
\Omega(r)\left(D^{2}-m^{2}\right) \tilde{\phi}(r)+2\left(D \hat{n}_{0}\right) \tilde{\phi}(r)=0,
$$

or

$$
\Omega(r)\left(D^{2}-m^{2}\right) \tilde{\phi}(r)-\tilde{\phi}(r) D(D+2) \Omega(r)=0,
$$

the quantity in brackets in the second equation being a known function. Introducing $\psi$ through $\tilde{\phi}=r^{m} \psi$, and noting that $D\left(r^{m} f\right)=r^{m}(D+m) f$, we obtain the pretty equation,

$$
\Omega(r) D(D+2 m) \psi(r)=\psi(r) D(D+2) \Omega(r) .
$$

At once, if $m=1$, we find $\psi(r)=A \Omega(r)$, or $\tilde{\phi}(r)=A r \Omega(r)$ $=A r\left[\omega-\hat{\omega}_{0}(r)\right]$. This simple $\tilde{\phi}(r)$ is a point eigenfunction, corresponding to $\omega=\hat{\omega}_{0}(b)$. The spectrum consists of a point sitting on the edge of a continuous spectrum, the latter coinciding with the set of values assumed by the function $\hat{\omega}_{0}(r)$. In this case the linear response cannot show simple sinusoidal behavior. Further, when $m=1$ the initial-value problem for the time-dependent equation may be integrated simply. ${ }^{8}$

The rather abstract form given for the differential equation makes obvious the possibility of analytical modeling. Merely write

$$
\frac{1}{\psi(r)} D(D+2 m) \psi(r)=\frac{1}{\Omega(r)} D(D+2) \Omega(r)=\mu(r),
$$

with $\mu(r)$ a simple, but arbitrary function, which permits solutions in terms of known functions. Then, a simultaneous solution of the two differential equations, under boundary and "positivity" conditions will yield equilibrium profiles, perturbed distributions, and point eigenvalues. The positivity condition is

$\hat{n}_{0}=\frac{1}{2}(D+2) \hat{\omega}_{0}(r) \geqslant 0, \quad \hat{\omega}_{0}(r)=\omega-\Omega(r), \quad$ for all $r$.

As an example, take $\mu(r)=c_{1} r /\left(1+c_{2} r\right)$. Then, both $\psi(r)$ and $\Omega(r)$ satisfy the hypergeometric equation, power-law frequency profiles being a special case. One then attempts to choose the constants so that boundary conditions and positivity are satisfied. The result, finally, is an equation for $\omega$. The exercise may be carried out just as easily for functions of $u=r^{2 p}$.

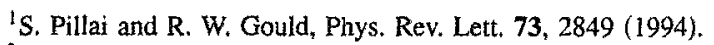

${ }^{2}$ J. S. deGrassie and J. H. Malmberg, Phys. Fluids 23, 63 (1980).

${ }^{3}$ R. J. Briggs, J. D. Daugherty, and R. H. Levy, Phys. Fluids 13, 421 (1970).

4J. D. Jackson, Classical Electrodynamics (Wiley, New York, 1962), p. 224.

${ }^{5}$ W. D. White, J. H. Malmberg, and C. F. Driscoll, Phys. Rev. Lett. 49, 1822 (1982).

${ }^{6}$ C. F. Driscoll and K. S. Fine, Phys. Fluids B 2, 1359 (1990).

${ }^{7}$ E. G. Broadbent and D. W. Moore, Proc. R. Soc. London Ser. A 384, 1 (1982).

${ }^{8}$ R. A. Smith and M. N. Rosenbluth. Phys. Rev. Lett, 64, 649 (1990). 